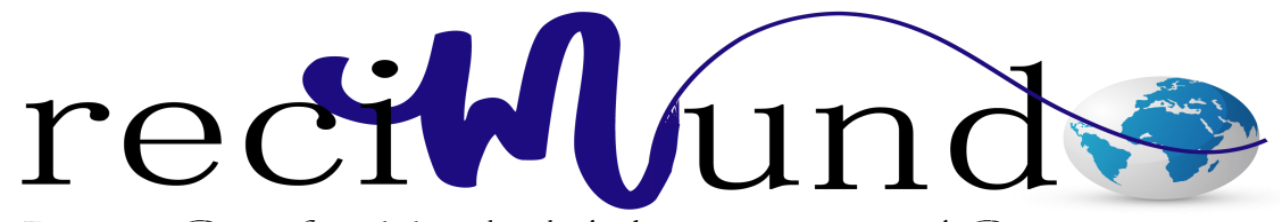

Revista Científica Mundo de la Investigación y el Conocimiento

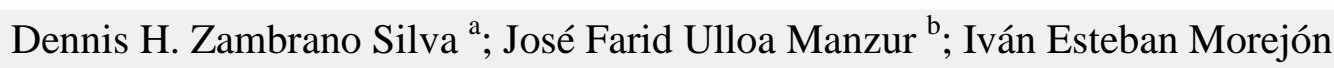
Coba $^{\text {c}}$; Mario Pinos Guerra ${ }^{\mathrm{d}}$

Modelo de inventario para el control económico de pedidos en Microempresa de Calzado

Revista Científica Mundo de la Investigación y el Conocimiento. Vol. 2 núm.2, mayo, ISSN: 2588-073X, 2018, pp. 566-584

DOI: 10.26820/recimundo/2.(2).2018.566-584

Editorial Saberes del Conocimiento

Recibido: 05/12/2017

Aceptado: 15/02/2018
a. Universidad de Guayaquil.
b. Universidad de Guayaquil.
c. Universidad de Guayaquil.
d. Universidad de Guayaquil. 
Modelo de inventario para el control económico de pedidos en Microempresa

de Calzado

Vol. 2, núm. 2., (2018)

Dennis H. Zambrano Silva; José Farid Ulloa Manzur; Iván Esteban Morejón Coba; Mario Pinos

Guerra

\section{RESUMEN}

El presente trabajo, propone la implementación de un modelo de inventarios en una microempresa de calzados en la ciudad de Guayaquil; aplicando este modelo es posible disminuir los costos de inventario y aumentar los beneficios financieros para la organización, mediante la rotación adecuada de las existencias empleando la mínima inversión posible. El procedimiento empleado se sustenta en la clasificación de los productos, considerando las variables de costo y la rotación de los modelos de mayor salida. La herramienta acorde a nuestros requerimientos es el método $\mathrm{ABC}$, el mismo que considera la magnitud económica de las ventas de cada producto para de esta manera determinar si el artículo pertenece a la clasificación, A, B, C. Por medio del modelo de la cantidad económica a pedir (CEP), se da seguimiento a productos vendidos, y se determina la cantidad óptima a pedir, la frecuencia para realizar un pedido y el punto mínimo de reabastecimiento, para su posterior control y toma de decisiones.

Palabras clave: Análisis de inventario ABC, Cantidad económica de pedido, Gestión de inventarios, Control de Inventarios. 


\title{
Modelo de inventario para el control económico de pedidos en Microempresa de Calzado
}

Vol. 2, núm. 2., (2018)

Dennis H. Zambrano Silva; José Farid Ulloa Manzur; Iván Esteban Morejón Coba; Mario Pinos Guerra

\begin{abstract}
The present work proposes the implementation of an inventory model for a small footwear company in the city of Guayaquil; applying this model, it is possible to reduce inventory costs and increase the financial benefits for the organization, through the adequate rotation of stocks using the minimum possible investment. The used procedure is based on the classification of the products, considering the variable costs and the rotation of the shoes' models with the highest sales. The tool according to our requirements is the ABC method, which considers the economic magnitude of each product sales to determine if the item belongs to the classification $\mathrm{A}, \mathrm{B}, \mathrm{C}$. Within the Quantity Economic Model Order by its initials in Spanish (CEP), the product that has been sold is tracked and it determines the optimal amount to order, the frequency to place an order and the minimum point of replenishment, for further control and decision making.
\end{abstract}

Key words: Inventory analysis $\mathrm{ABC}$, economic quantity of order, management of inventories, Control of inventories. 


\section{Modelo de inventario para el control económico de pedidos en Microempresa}

de Calzado

Vol. 2, núm. 2., (2018)

Dennis H. Zambrano Silva; José Farid Ulloa Manzur; Iván Esteban Morejón Coba; Mario Pinos

Guerra

\section{Introducción.}

En los últimos años el interés por parte de muchas microempresas se ha maximizado en lo que respecta al control y la gestión de inventarios, ya que este constituye una ventaja competitiva ya que no se puede afectar la disponibilidad de los productos, en otra palabras, si la organización no cuenta con el producto que se necesita para suplir el pedido solicitado se puede llegar a perder clientes, es decir, y esto no es conveniente, puesto que la satisfacción de estos representa lo más importante para la organización. De manera antagónica ocurre que, si se cuenta con demasiada materia prima, producto en proceso y producto terminado almacenados en bodega (stock) la organización experimenta un aumento en los costos de mantener ese inventario, lo que conlleva a que se generen desperdicios, los mismos que, desagregan valor al proceso, producto y/o servicio, para evitar aquello es indispensable que la organización cuente con un correcto control y gestión en sus inventarios. (Taboada González, Aguilar Virgen, Ibarra Trujillo, \& Ramírez Barreto, 2016)

La gestión de inventarios es un sistema transversal a la cadena de abastecimiento, que se relaciona con la planificación y el debido control de los inventarios y busca decidir entre ¿Cuándo Pedir? ¿Cuánto Pedir? (Quintana, 2010, págs. 13, 14).

Entre los beneficios que implica el aplicar un sistema de Gestión de Inventarios incluye el reducir los costos y mejorar la eficiencia económica, ya que incrementa los niveles de servicio al cliente, aumenta la liquidez y permite a las organizaciones estar prevenidas frente a las fluctuaciones de la demanda; manteniendo un óptimo nivel de seguridad y logrando mantener los 


\section{Modelo de inventario para el control económico de pedidos en Microempresa de Calzado}

Vol. 2, núm. 2., (2018)

Dennis H. Zambrano Silva; José Farid Ulloa Manzur; Iván Esteban Morejón Coba; Mario Pinos Guerra

inventarios necesarios del producto. (Pérez Vergara, Cifuentes Laguna, Vásquez García , \& Ocampo, 2013)

Según lo afirmado por Heizer \& Render (2009), "El análisis ABC divide el inventario disponible en tres clases $(\mathrm{a}, \mathrm{b}, \mathrm{c})$ con base en su volumen anual en dinero, esta es una aplicación a los inventarios de lo que se conoce como principio de Pareto, el mismo establece que hay "pocos artículos cruciales y muchos triviales", ya que no es realista monitorear los artículos baratos con la misma intensidad que a los artículos costosos" (pág. 485)

Particularmente en este artículo se busca desarrollar un modelo de inventario para el control económico de pedidos en Microempresa de Calzado "BAZKING FOOTWEAR" con el objeto de determinar la cantidad óptima de producto que se debe pedir y así mismo reducir los costos de manejo del inventario que se tiene actualmente mediante la aplicación del modelo de Cantidad Económica de Pedido (CEP).

\section{Materiales y métodos}

La metodología a seguir incluye la aplicación del método $\mathrm{ABC}$ el mismo divide los artículos en la clase "A" que constituyen sólo un $15 \%$ de todos los artículos del inventario, pero representan entre el $70 \%$ y el $80 \%$ del uso total en dinero, estos a su vez exigen un tratamiento detallado, muchos clientes lo piden y están acostumbrados a llevárselo; clase "B" representan alrededor del $30 \%$ de todo el inventario y entre un $15 \%$ y un $25 \%$ del valor total, tienen que ser también vigilados aunque con menor frecuencia, por último y sin dejar de lado se tiene los artículos de clase "C" estos constituyen sólo un 5\% de tal volumen pero casi el 55\% de los 


\section{Modelo de inventario para el control económico de pedidos en Microempresa de Calzado}

Vol. 2, núm. 2., (2018)

Dennis H. Zambrano Silva; José Farid Ulloa Manzur; Iván Esteban Morejón Coba; Mario Pinos

Guerra

artículos en inventario, para estos el control no debe ser muy frecuente. (Heizer \& Render, 2009).

El análisis del método ABC se aplicará en BAZKING FOOTWEAR para los ítems de producto terminado (zapatos para caballeros) y así poder determinar sobre qué grupo se hará el análisis; por consecuencia, se cuenta con los nombres de los artículos vendidos en BAZKING, precios unitarios y volumen de ventas en el año, tal como se muestra en la Tabla. 1.

Tabla 1. Volumen de ventas en el 2017

\begin{tabular}{|c|c|c|c|c|c|c|c|c|c|c|c|c|c|}
\hline 10 & $\Delta$ & (8) & & & $\begin{array}{r}\text { Cantida } \\
\text { BAZ }\end{array}$ & $\begin{array}{r}\text { ad de } P \\
\text { ZKING F } \\
\text { Año }\end{array}$ & $\begin{array}{l}\text { ares V } \\
=00 T \text { Th } \\
2017\end{array}$ & $\begin{array}{l}\text { Jendidos } \\
\text { VEAR }\end{array}$ & & & & & \\
\hline $\begin{array}{l}\text { Código del } \\
\text { Artículo }\end{array}$ & ENERO & FEBRERO & MARZO & ABRIL & MAYO & JUNIO & JULIO & AGOSTO & SEPTIEMBRE & OCTUBRE & NOVIEMBRE & DICIEMBRE & TOTAL \\
\hline Botas $4 \times 4$ todo terreno & 39 & 37 & 37 & 37 & 38 & 39 & 39 & 40 & 38 & 38 & 39 & 36 & 457 \\
\hline Douglas Deportivo & 10 & 9 & 11 & 8 & 10 & 13 & 11 & 13 & 10 & 11 & 12 & 11 & 129 \\
\hline Patrick Botin Deportivo & 11 & 10 & 12 & 12 & 10 & 13 & 11 & 12 & 10 & 9 & 10 & 11 & 131 \\
\hline Casual Sebastián & 31 & 30 & 31 & 33 & 30 & 27 & 32 & 31 & 33 & 29 & 30 & 32 & 369 \\
\hline Casual Arnold & 36 & 41 & 37 & 43 & 37 & 43 & 39 & 38 & 42 & 41 & 43 & 45 & 485 \\
\hline Casual Brandon & 39 & 35 & 35 & 33 & 36 & 33 & 35 & 34 & 32 & 34 & 31 & 30 & 407 \\
\hline Casual Iker & 41 & 40 & 40 & 42 & 38 & 44 & 45 & 36 & 40 & 35 & 35 & 40 & 476 \\
\hline Charles Deportivo & 10 & 10 & 9 & 9 & 8 & 9 & 9 & 10 & 9 & 9 & 10 & 11 & 113 \\
\hline Casual Ariel & 39 & 45 & 35 & 43 & 40 & 37 & 42 & 45 & 46 & 43 & 48 & 37 & 500 \\
\hline Casual Danilo & 38 & 41 & 40 & 38 & 39 & 40 & 38 & 43 & 38 & 45 & 48 & 46 & 494 \\
\hline Gabriel Deportivo & 12 & 10 & 9 & 11 & 8 & 7 & 9 & 10 & 9 & 9 & 10 & 11 & 115 \\
\hline Jacob Deportivo & 10 & 10 & 9 & 9 & 8 & 9 & 9 & 11 & 9 & 9 & 10 & 11 & 114 \\
\hline Edward Deportivo & 6 & 9 & 9 & 8 & 7 & 9 & 10 & 9 & 9 & 10 & 10 & 9 & 105 \\
\hline Urban style & 16 & 15 & 19 & 20 & 21 & 14 & 19 & 23 & 18 & 15 & 16 & 18 & 214 \\
\hline Casual Leonidas & 6 & 8 & 6 & 8 & 8 & 7 & 7 & 9 & 6 & 9 & 8 & 7 & 89 \\
\hline Casual Andy & 44 & 45 & 47 & 45 & 48 & 46 & 43 & 44 & 49 & 50 & 49 & 48 & 558 \\
\hline Omar Deportivo & 13 & 16 & 15 & 12 & 10 & 15 & 11 & 14 & 9 & 9 & 10 & 11 & 145 \\
\hline Maurice Deportivo & 6 & 9 & 9 & 8 & 8 & 9 & 10 & 9 & 8 & 9 & 10 & 9 & 104 \\
\hline Jordan Deportivo & 11 & 10 & 12 & 12 & 10 & 13 & 11 & 12 & 13 & 9 & 10 & 11 & 134 \\
\hline
\end{tabular}

Fuente: Datos proporcionados por BAZKING FOOTWEAR 


\section{Modelo de inventario para el control económico de pedidos en Microempresa de Calzado}

Vol. 2, núm. 2., (2018)

Dennis H. Zambrano Silva; José Farid Ulloa Manzur; Iván Esteban Morejón Coba; Mario Pinos Guerra

Posterior a ello, se desarrollará el modelo básico de la cantidad económica a pedir (CEP), también denominado EOQ por sus siglas en inglés, el mismo consiste en amenorar los costos totales de ordenar y mantener el inventario, además, constituye un método fácil para el control de inventarios, por otro lado, para contar con información relativa a la demanda se tomó como referencia las ventas que hubo en el año 2017 para tal efecto. (Rodríguez, 2015)

Según el libro Principios de Administración de Operaciones escrito por (Heizer \& Render, 2009), se dice que la cantidad económica a pedir se basa en los siguientes supuestos: "La demanda del producto (D) es conocida, constante e independiente, el tiempo de entrega es conocido y constante, $\mathrm{El}$ inventario se resurte al momento en el que inventario existente llega a cero o cuando llega un lote de pedido, los descuentos por cantidad no son posibles y los faltantes (inexistencias) se evitan por completo si las órdenes se colocan en el momento correcto" (pág. 490).

Para la comprensión de la simbología utilizada se tienen las siguientes aclaraciones, tal como sigue:

$Q=$ Número de unidades

$\mathbf{Q}^{*}=$ Cantidad óptima de unidades a pedir (CEP)

$\mathbf{D}=$ Demanda anual en unidades

$\mathbf{S}=$ Costo unitario de ordenar para cada pedido $(\$)$

$\mathbf{h}=\%$ de costo de mantener el inventario 


\section{Modelo de inventario para el control económico de pedidos en Microempresa}

de Calzado

Vol. 2, núm. 2., (2018)

Dennis H. Zambrano Silva; José Farid Ulloa Manzur; Iván Esteban Morejón Coba; Mario Pinos

Guerra

$\mathbf{C}=$ Costo unitario del producto $(\$)$

$\mathbf{N}=$ Frecuencia de pedido anual

$\mathbf{R}=$ Punto de reorden

$\mathbf{L}=$ tiempo de espera (días)

Por consiguiente, es importante conocer cuáles son los costos totales, estos constituyen la adición del costo total de ordenar y el costo total de mantener inventario, para ello primero se debe determinar la cantidad económica a pedir, siendo sus fórmulas respectivas las siguientes:

$$
Q^{*}=\sqrt{\frac{2 D S}{h * c}} \quad ; \quad S_{T}=\frac{D}{Q^{*}}(S) \quad ; \quad H_{T}=\frac{Q^{*}}{2}(h * c)
$$

Según lo Afirmado por Chopra \& Meindl (2008) se dice que : "Los costos totales de ordenar y mantener inventario son relativamente estables alrededor de la cantidad económica de pedido. A la compañía le conviene más ordenar un tamaño de lote práctico, cercano a la cantidad económica de pedido, en lugar del valor exacto de la CEP” (pág. 267)

Es indispensable saber con qué regularidad se deben realizar los pedidos para poder abastecer satisfactoriamente a la demanda, sumado a esto es necesario que BAZKING FOOTWEAR conozca en qué punto se debe resurtir o reabastecer su bodega, esto mediante el punto de reorden, para tal efecto se tienen las siguientes fórmulas para su posterior cálculo y análisis. 


\section{Modelo de inventario para el control económico de pedidos en Microempresa de Calzado}

Vol. 2, núm. 2., (2018)

Dennis H. Zambrano Silva; José Farid Ulloa Manzur; Iván Esteban Morejón Coba; Mario Pinos Guerra

$$
N=\frac{D}{Q^{*}} \quad ; \quad R=D * L
$$

Cabe aclarar que para hallar el punto de reorden " $R$ ", se debe considerar la demanda diaria y no la anual, dado esto, por último, se tiene la realización del diseño de las políticas de inventario para la microempresa BAZKING FOOTWEAR.

\section{Resultados}

Aplicación del Análisis ABC

Con base a la información obtenida en la Tabla 1. Es posible empezar con la aplicación del método ABC, para ello se averiguaron los precios unitarios de los productos producidos y comercializados en Bazking, luego se calculó el total vendido anualmente (\$) para cada producto, posterior a ello se calculó el porcentaje de participación y el porcentaje acumulado de dicha participación para así lograr clasificar a los artículos en Clase A, B, C., todo esto, en base a los criterios establecidos anteriormente. Tabla 2. 


\section{Modelo de inventario para el control económico de pedidos en Microempresa}

de Calzado

Vol. 2, núm. 2., (2018)

Dennis H. Zambrano Silva; José Farid Ulloa Manzur; Iván Esteban Morejón Coba; Mario Pinos

Guerra

\section{Tabla 2 Análisis ABC en BAZKING FOOOTWEAR}

\begin{tabular}{|r|l|c|c|c|c|c|c|}
\hline No. & \multicolumn{1}{|c|}{$\begin{array}{c}\text { Código del } \\
\text { Artículo }\end{array}$} & $\begin{array}{c}\text { Cantidad de } \\
\text { Pares } \\
\text { vendidos }\end{array}$ & PVP & Total Vendido $\$$ (\$) & Participacion & Participación \\
Acumulada & Clasificación \\
\hline 1 & Casual Danilo & 494 & $\$ 58.00$ & $\$ 28,652.00$ & $13.82 \%$ & $13.82 \%$ & $\mathrm{~A}$ \\
\hline 2 & Botas 4x4 todo terreno & 457 & $\$ 60.00$ & $\$ 27,420.00$ & $13.23 \%$ & $27.05 \%$ & $\mathrm{~A}$ \\
\hline 3 & Casual Arnold & 485 & $\$ 55.00$ & $\$ 26,675.00$ & $12.87 \%$ & $39.92 \%$ & $\mathrm{~A}$ \\
\hline 4 & Casual Andy & 558 & $\$ 45.00$ & $\$ 25,110.00$ & $12.11 \%$ & $52.04 \%$ & $\mathrm{~A}$ \\
\hline 5 & Casual Ariel & 500 & $\$ 46.00$ & $\$ 23,000.00$ & $11.10 \%$ & $63.13 \%$ & $\mathrm{~A}$ \\
\hline 6 & Casual Iker & 476 & $\$ 48.00$ & $\$ 22,848.00$ & $11.02 \%$ & $74.16 \%$ & $\mathrm{~A}$ \\
\hline 7 & Casual Sebastián & 369 & $\$ 38.00$ & $\$ 14,022.00$ & $6.77 \%$ & $80.92 \%$ & $\mathrm{~B}$ \\
\hline 8 & Casual Brandon & 407 & $\$ 30.00$ & $\$ 12,210.00$ & $5.89 \%$ & $86.81 \%$ & $\mathrm{~B}$ \\
\hline 9 & Urban style & 214 & $\$ 18.00$ & $\$ 3,852.00$ & $1.86 \%$ & $88.67 \%$ & $\mathrm{~B}$ \\
\hline 10 & Omar Deportivo & 145 & $\$ 23.00$ & $\$ 3,335.00$ & $1.61 \%$ & $90.28 \%$ & $\mathrm{~B}$ \\
\hline 11 & Gabriel Deportivo & 115 & $\$ 25.00$ & $\$ 2,875.00$ & $1.39 \%$ & $91.67 \%$ & $\mathrm{~B}$ \\
\hline 12 & Charles Deportivo & 113 & $\$ 25.00$ & $\$ 2,825.00$ & $1.36 \%$ & $93.03 \%$ & $\mathrm{~B}$ \\
\hline 13 & Douglas Deportivo & 129 & $\$ 19.00$ & $\$ 2,451.00$ & $1.18 \%$ & $94.21 \%$ & $\mathrm{~B}$ \\
\hline 14 & Patrick Botin Deportivo & 131 & $\$ 18.00$ & $\$ 2,358.00$ & $1.14 \%$ & $95.35 \%$ & $\mathrm{~B}$ \\
\hline 15 & Jordan Deportivo & 134 & $\$ 17.00$ & $\$ 2,278.00$ & $1.10 \%$ & $96.45 \%$ & $\mathrm{C}$ \\
\hline 16 & Edward Deportivo & 105 & $\$ 20.00$ & $\$ 2,100.00$ & $1.01 \%$ & $97.46 \%$ & $\mathrm{C}$ \\
\hline 17 & Casual Leonidas & 89 & $\$ 20.00$ & $\$ 1,780.00$ & $0.86 \%$ & $98.32 \%$ & $\mathrm{C}$ \\
\hline 18 & Maurice Deportivo & 104 & $\$ 17.00$ & $\$ 1,768.00$ & $0.85 \%$ & $99.17 \%$ & $\mathrm{C}$ \\
\hline 19 & Jacob Deportivo & 114 & $\$ 15.00$ & $\$ 1,710.00$ & $0.83 \%$ & $100.00 \%$ & $\mathrm{C}$ \\
\hline & & & $\$ 207,269.00$ & $100.00 \%$ & & \\
\hline
\end{tabular}

Fuente: Cálculos realizados por el autor

Dada la realización de la clasificación ABC para BAZKING FOOTWEAR y teniendo en cuenta que los artículos más importantes para ellos son los de tipo A, debido al porcentaje de participación en ventas que representan, se muestran los siguientes resultados: 
Modelo de inventario para el control económico de pedidos en Microempresa de Calzado

Vol. 2, núm. 2., (2018)

Dennis H. Zambrano Silva; José Farid Ulloa Manzur; Iván Esteban Morejón Coba; Mario Pinos Guerra

Tabla 3 Porcentaje de Participación en Ventas para el inventario ABC

\begin{tabular}{|c|c|c|c|c|c|}
\hline CLASE & $\%$ & CANTIDAD & \% PARTICIPACION & VENTAS & $\begin{array}{c}\text { PARTICIPACION EN } \\
\text { VENTAS }\end{array}$ \\
\hline A & $0-80 \%$ & 6 & $31.58 \%$ & $\$ 153,705.00$ & $74.16 \%$ \\
\hline B & $81 \%-95 \%$ & 8 & $42.11 \%$ & $\$ 43,928.00$ & $21.19 \%$ \\
\hline C & $96 \%-100 \%$ & 5 & $26.32 \%$ & $\$ 9,636.00$ & $4.65 \%$ \\
\hline \multicolumn{2}{|c|}{ TOTAL } & 19 & $100.00 \%$ & $\$ 207,269.00$ & $100.00 \%$ \\
\hline
\end{tabular}

Fuente: Cálculos realizados por el autor

Gráfico 1 Clasificación ABC Bazking Footwear

\section{ANALISIS ABC BAZKING FOOTWEAR}

$80,00 \%$

$70,00 \%$

$60,00 \%$

$50,00 \%$

$40,00 \%$

$30,00 \%$

$20,00 \%$

$10,00 \%$

$0,00 \%$

\section{$74,16 \%$}

$21,19 \%$

$4,65 \%$

A

B

C

Elaborado: Por el autor

Aplicación del modelo de inventario Cantidad Económica a Pedir (CEP) 
Modelo de inventario para el control económico de pedidos en Microempresa de Calzado

Vol. 2, núm. 2., (2018)

Dennis H. Zambrano Silva; José Farid Ulloa Manzur; Iván Esteban Morejón Coba; Mario Pinos

Guerra

Según (Departamento de Organización de Empresas, 2013): "Los artículos de clase A exigen un tratamiento detallado, muchos clientes lo piden y están acostumbrados a llevárselo, quedarse sin stock tiene repercusiones importantes. En estos artículos el control de existencias debe ser frecuente, así como la evaluación de las previsiones.” (pág. 10)

Ahora bien, para determinar la cantidad óptima a pedir primero se procede a seleccionar la demanda anual con la que cuentan los artículos clasificados como tipo A.

Tabla 4 Demanda Anual para los artículos de clase A

\begin{tabular}{|c|c|}
\hline PRODUCTOS & $\begin{array}{c}\text { DEMANDA ANUAL } \\
\text { 2017 }\end{array}$ \\
\hline Casual Danilo & 494 \\
\hline Botas 4x4 todo terreno & 457 \\
\hline Casual Arnold & 485 \\
\hline Casual Andy & 558 \\
\hline Casual Ariel & 500 \\
\hline Casual Iker & 476 \\
\hline
\end{tabular}

Datos proporcionados por BAZKING FOOTWEAR 


\section{Modelo de inventario para el control económico de pedidos en Microempresa}

de Calzado

Vol. 2, núm. 2., (2018)

Dennis H. Zambrano Silva; José Farid Ulloa Manzur; Iván Esteban Morejón Coba; Mario Pinos Guerra

Costo total del pedido

Para calcular el costo total del pedido se procede a seleccionar los pares de zapatos vendidos en un determinado mes, en este caso se ha considerado el mes de diciembre, además, se considera el costo unitario de cada uno de estos artículos, por último, se realiza un producto de ambos. Ver Tabla 5.

Tabla 5 Costo Total de Pedido para artículos clase A

\begin{tabular}{|l|c|c|c|}
\hline DESCRIPCIÓN & $\begin{array}{c}\text { PARES } \\
\text { POR } \\
\text { MES }\end{array}$ & $\begin{array}{c}\text { COSTO } \\
\text { UNITARIO } \\
\text { DEL } \\
\text { PRODUCTO } \\
\text { c }\end{array}$ & $\begin{array}{c}\text { COSTO } \\
\text { TOTAL } \\
\text { DEL } \\
\text { PEDIDO }\end{array}$ \\
\hline Casual Danilo & 46 & $\$ 58.00$ & $\$ 2,668.00$ \\
\hline Botas 4x4 todo terreno & 36 & $\$ 60.00$ & $\$ 2,160.00$ \\
\hline Casual Arnold & 45 & $\$ 55.00$ & $\$ 2,475.00$ \\
\hline Casual Andy & 48 & $\$ 45.00$ & $\$ 2,160.00$ \\
\hline Casual Ariel & 37 & $\$ 46.00$ & $\$ 1,702.00$ \\
\hline Casual Iker & 40 & $\$ 48.00$ & $\$ 1,920.00$ \\
\hline
\end{tabular}

Fuente: Cálculos realizados por el autor

Costo de mantener el inventario

La microempresa Bazking, como ya se dijo al inicio de este artículo, no cuenta con ningún control en sus inventarios, por tanto, no cuenta con información relaciona a la fracción de interés para el manejo del inventario, para ello se recurre al criterio expuesto por Vidal H., (2010), el mismo que expone que " por conceptos de interés y costo de oportunidad (4 \%), 
Modelo de inventario para el control económico de pedidos en Microempresa de Calzado

Vol. 2, núm. 2., (2018)

Dennis H. Zambrano Silva; José Farid Ulloa Manzur; Iván Esteban Morejón Coba; Mario Pinos

Guerra

obsolescencia y depreciación $(1,5 \%)$, almacenamiento y manejo (1,5\%), impuestos $(0,5 \%)$ y seguros $(0,5 \%)$, se tiene una tasa de interés para manejo del inventario del $8 \%$ sobre el valor del producto dado que, a cierto tiempo de haberlo pedido este se deteriora y se pierde;" Por tanto, para el cálculo del costo de mantener inventario se toma el $8 \%$ (h) como referencia. Ver Tabla 6.

Tabla 6 Costo de Mantener el Inventario tipo A

\begin{tabular}{|l|c|c|}
\hline PRODUCTO & $\begin{array}{c}\text { COSTO } \\
\text { DEL PRODUCTO } \\
\mathbf{c}\end{array}$ & $\begin{array}{c}\text { COSTO } \\
\text { UNITARIO DE } \\
\text { MANTENER } \\
\text { INVENTARIO } \\
\text { h*c }\end{array}$ \\
\hline Casual Danilo & $\$ 58.00$ & $\$ 4.64$ \\
\hline $\begin{array}{l}\text { Botas 4x4 todo } \\
\text { terreno }\end{array}$ & $\$ 60.00$ & $\$ 4.80$ \\
\hline Casual Arnold & $\$ 55.00$ & $\$ 4.40$ \\
\hline Casual Andy & $\$ 45.00$ & $\$ 3.60$ \\
\hline Casual Ariel & $\$ 46.00$ & $\$ 3.68$ \\
\hline Casual Iker & $\$ 48.00$ & $\$ 3.84$ \\
\hline
\end{tabular}

Fuente: Cálculos realizados por el autor

Costo de ordenar un pedido

Para determinar este costo, se considera lo aplicado por Rodríguez (2015), el mismo sostiene que: "la tasa de interés de ordenar es del $4.5 \%$ sobre el valor total del pedido" (pág. 174), dado esto se procede a realizar dichos cálculos, tal como sigue: Ver Tabla 7. 
Modelo de inventario para el control económico de pedidos en Microempresa de Calzado

Vol. 2, núm. 2., (2018)

Dennis H. Zambrano Silva; José Farid Ulloa Manzur; Iván Esteban Morejón Coba; Mario Pinos Guerra

Tabla 7. Costo De ordenar un pedido

\begin{tabular}{|l|c|c|c|}
\hline PRODUCTO & $\begin{array}{c}\text { CANTIDAD } \\
\text { (pares) }\end{array}$ & VALOR TOTAL (\$) & $\begin{array}{c}\text { COSTO DEL PEDIDO } \\
\text { (S* Valor Total) }\end{array}$ \\
\hline Casual Danilo & 46 & $\$ 2,668.00$ & $\$ 120.06$ \\
\hline Botas 4x4 todo terreno & 36 & $\$ 2,160.00$ & $\$ 97.20$ \\
\hline Casual Arnold & 45 & $\$ 2,475.00$ & $\$ 111.38$ \\
\hline Casual Andy & 48 & $\$ 2,160.00$ & $\$ 97.20$ \\
\hline Casual Ariel & 37 & $\$ 1,702.00$ & $\$ 76.59$ \\
\hline Casual Iker & 40 & $\$ 1,920.00$ & $\$ 86.40$ \\
\hline
\end{tabular}

Fuente: Cálculos realizados por el autor

En último lugar, se realiza el cálculo de la cantidad económica de pedido $\left(\mathrm{Q}^{*}\right)$ para los catorce artículos clasificados como A, además también se calcula el costo total de mantener y ordenar un pedido, la frecuencia con la que se realiza un pedido anualmente, el lead time, que constituye el tiempo de espera entre el momento en que se realiza un pedido y se lo recibe, también el punto de reorden que indica el momento en unidades en el cual es necesario realizar un reabastecimiento de esos productos. Ver Tabla 8.

En términos de aclaración, para calcular el tiempo de espera se considera un total de 298 días laborables hábiles dentro del 2017 sobre la frecuencia de pedido anual, además, para hallar el punto de reorden se tomó en cuenta la demanda diaria de cada uno de los artículos, esto se lo 
Modelo de inventario para el control económico de pedidos en Microempresa de Calzado

Vol. 2, núm. 2., (2018)

Dennis H. Zambrano Silva; José Farid Ulloa Manzur; Iván Esteban Morejón Coba; Mario Pinos

Guerra

realizó mediante la división entre la demanda anual de cada producto y el número de días laborables en Bazking.

Tabla 8. Cantidad Económica a Pedir para artículos clase A

\begin{tabular}{|l|c|c|c|c|c|c|}
\hline PRODUCTO & $\begin{array}{c}\text { DEMAND } \\
\text { A } \\
\text { D }\end{array}$ & $\begin{array}{c}\text { CEP } \\
\left(\mathbf{Q}^{*}\right)\end{array}$ & $\begin{array}{c}\text { COSTO } \\
\text { TOTAL DE } \\
\text { INVENTARI } \\
\text { O } \\
\text { CT }\end{array}$ & $\begin{array}{c}\text { FRECUENCI } \\
\text { A DE } \\
\text { PEDIDO } \\
(\text { AÑ }) \\
\text { N })\end{array}$ & $\begin{array}{c}\text { TIEMP } \\
\text { O DE } \\
\text { ESPER } \\
\text { A L } \\
\text { días) }\end{array}$ & $\begin{array}{c}\text { PUNTO } \\
\text { DE } \\
\text { REORDE } \\
\text { N R } \\
\mathbf{d}^{*} \mathbf{L}\end{array}$ \\
\hline Casual Danilo & 494 & 159.889 & $\$ 124.70$ & 3.090 & 96 & 160 \\
\hline $\begin{array}{l}\text { Botas 4x4 todo } \\
\text { terreno }\end{array}$ & 457 & 136.046 & $\$ 102.00$ & 3.359 & 89 & 136 \\
\hline Casual Arnold & 485 & 156.694 & $\$ 115.78$ & 3.095 & 96 & 157 \\
\hline Casual Andy & 558 & 173.586 & $\$ 100.80$ & 3.215 & 93 & 174 \\
\hline Casual Ariel & 500 & 144.265 & $\$ 80.27$ & 3.466 & 86 & 144 \\
\hline Casual Iker & 476 & 146.356 & $\$ 90.24$ & 3.252 & 92 & 146 \\
\hline
\end{tabular}

Fuente: Cálculos realizados por el autor

\section{Discusión.}

En base a los resultados conseguidos a través de la aplicación del analisis ABC en LA Microempresa de Calzado BAZKING FOOTWEAR se obtiene como resultado que, dentro de los artículos clasificados como tipo A, están seis categorías de zapatos, ya que estos representan el $74.16 \%$ del volumen de ventas, por tanto, los mismos constituyen los productos más beneficiosos financieramente para la microempresa, como se observa en los cuadros anteriores, al establecer el modelo de zapato tipo "A" se incluyen todas las tallas posibles de compra, por tanto este detalle debe ser tomado en cuenta para que al momento de su elaboración se fabriquen 


\section{Modelo de inventario para el control económico de pedidos en Microempresa de Calzado}

Vol. 2, núm. 2., (2018)

Dennis H. Zambrano Silva; José Farid Ulloa Manzur; Iván Esteban Morejón Coba; Mario Pinos Guerra

la mayor cantidad de pares de las tallas de más salida. Consecuentemente a esto, se tiene que a dichos artículos en el momento de realizar los inventarios respectivos se le tiene que realizar un mayor monitoreo.

Respecto a los resultados arrojados por la cantidad económica a pedir, se evidencian las demandas anuales respectivas a cada producto clasificado como A, sumado a esto también se exhiben los costos totales anuales en los que se incurren al momento de ordenar un pedido, el costo total anual de mantenerlos, la frecuencia o regularidad con la que se realizan los pedidos en términos anuales, además el punto de reorden el cual indica en qué momento se deben resurtir las bodegas, como por ejemplo el Casual Danilo al llegar a 160 pares en bodega debe resurtirse esto en un plazo no mayor a 96 días como lo indica su respectivo lead time, con el objeto de no desabastecer a la demanda de ese producto.

Por otro lado, se tiene que el calzado Andy tiene el valor más alto de demanda dentro de todos los artículos de clase A, está representado por una demanda de 558 pares y al instante de realizar un pedido para este artículo Bazking incurre en un costo de \$97.20, además, otro costo asociado a mantener ese producto en inventario es de $\$ 3.60$ mensualmente así mismo, para concluir se tiene que la cantidad económica a pedir mensual es de 174 pares de calzado Andy.

En otras palabras, se cumple la condición de que el inventario se resurte al momento en el que inventario existente llega a cero, ya que el punto de reorden también equivale a 174 pares, los cinco artículos restantes son objetos del mismo análisis según la conveniencia del lector. 


\section{Modelo de inventario para el control económico de pedidos en Microempresa}

de Calzado

Vol. 2, núm. 2., (2018)

Dennis H. Zambrano Silva; José Farid Ulloa Manzur; Iván Esteban Morejón Coba; Mario Pinos

Guerra

\section{Conclusiones y recomendaciones.}

La implementación de un software que facilite el manejo de los datos y de las existencias junto con sus costos unitarios es de enorme importancia, para acelerar los cálculos y retroalimentar el sistema constantemente. Los modelos que vayan cayendo en una rotación menor, deben colocarse como promociones o ajustar su precio de venta con el fin de recuperar rápidamente su inversión.

El modelo de inventarios CEP, constituye una herramienta de fácil manejo para el control de los inventarios y que además integra por ejemplo el punto de reorden de los pedidos, la frecuencia con la que se debe pedir y cada que tiempo pedir, Evidentemente, con este modelo la organización se asegura de ¿Cuándo Pedir? y ¿Cuánto Pedir a fin de implantar una política firme que permita mejorar los resultados?

Por lo antes mencionado, se recomienda a Bazking Footwear la implementación del modelo aplicado en el presente, dado que los beneficios, podrán verse cristalizados con la presencia de bajos costos ya sea a mediano o largo plazo. Sin embargo, cabe recalcar que el presente modelo solo ha considerado los artículos clasificados A, por lo cual, está sujeto a futuros cambios, en cuanto a la representación analítica y a los resultados obtenidos, en el caso de que la organización adopte este modelo para todos sus productos. 


\section{Modelo de inventario para el control económico de pedidos en Microempresa}

de Calzado

Vol. 2, núm. 2., (2018)

Dennis H. Zambrano Silva; José Farid Ulloa Manzur; Iván Esteban Morejón Coba; Mario Pinos Guerra

\section{Referencias Bibliográficas}

Chopra , S., \& Meindl, P. (2008). Administración de la cadena de suministro (Tercera ed.). México: Pearson Educación.

Departamento de Organización de Empresas, E. y. (2013). GESTIÓN DE INVENTARIOS DE DEMANDA INDEPENDIENTE. Mexico D.F., Mexico.

Heizer, J., \& Render, B. (2009). Principios de Administración de Operaciones. México: PEARSON EDUCACION.

Pérez Vergara , I., Cifuentes Laguna, A., Vásquez García , C., \& Ocampo, D. (2013). Un modelo de gestión de inventarios para una empresa de productos alimenticios. Ingeniería Industrial, $X X X I V(2), 227-236$.

Quintana, D. L. (Febrero de 2010). Propuesta de un Sistema de Gestión de Inventarios para una empresa comercializadora de productos Plásticos. Lima, Perú.

Rodríguez, E. C. (2015). Modelo de inventarios para control económico de pedidos en empresa Comercializadora de alimentos. Redalyc, 14(27), 163-177. Obtenido de http://www.redalyc.org/articulo.oa?id=75045730012

Taboada González, P., Aguilar Virgen, Q., Ibarra Trujillo, J., \& Ramírez Barreto, M. (2016). Optimización de un Sistema de Abastecimiento de pintura a concesionarios de baja y media demanda. Información Tecnólogica, 27(3), 53-60. doi:10.4067/S071807642016000300006

Vidal H., C. (2010). Fundamentos de Control y Gestión de Inventarios. Cali, Colombia: Universidad del Valle. 